\title{
Multi-Weight Unipolar Codes for Multimedia Spectral-Amplitude-Coding Optical CDMA Systems
}

\author{
Ivan B. Djordjevic, Bane Vasic, Senior Member, IEEE, and Judy Rorison
}

\begin{abstract}
A novel balanced detection scheme for multi-access interference cancellation in multimedia spectral-amplitude-coding optical code-division multiple-access system is proposed. The receiver is capable of handling both equal and unequal in-phase cross correlation of optical orthogonal codes. A novel class of constantlength variable-weight optical orthogonal codes able to support multimedia services with different quality-of-service requirements is proposed as well. The construction is based on the pairwise balanced designs, or more specifically, on an incidence structure defined on an integer sub-lattice.
\end{abstract}

Index Terms-Integer lattices, multi-access interference (MAI), multimedia, optical code division multiple access (CDMA), optical orthogonal codes, pairwise balanced designs, spectral-amplitudecoding (SAC).

\section{INTRODUCTION}

$\mathbf{T}$ HE main factor of performance degradation in optical code-division multiple-access (CDMA) systems is the multi-access interference (MAI) [1]-[7]. In spectral amplitude-coding (SAC) systems [1]-[3], MAI is solely a function of the in-phase cross correlations among the address sequences (also known as signature sequences or spreading sequence or simply code sequences). The existing balanced detection schemes for SAC systems, [3], require unipolar sequences having fixed in-phase cross correlation. Hadamard code, $m$-sequence, modified quadratic congruence code (MQC) and modified frequency hopping (MFH) codes have been proposed for such applications [1]-[3]. None of them is suitable for multimedia applications with different quality-of-service requirements.

We propose a balanced detection fiber Bragg grating scheme (FBG), capable of canceling the multi-user interference for SAC system employing unipolar codes with nonfixed in-phase cross correlations in multimedia environment.

A novel constant-length variable-weight class of optical orthogonal codes is proposed as well. The construction is based on combinatorial designs, more specifically on pairwise balanced

Manuscript received August 13, 2003. The associate editor coordinating the review of this letter and approving it for publication was Prof. D. P. Taylor This work is supported by the National Science Foundation under Grant ITR 0325979 .

I. B. Djordjevic was with University of Bristol, Bristol BS8 1TR, U.K. He is now with the Department of Electrical and Computer Engineering, University of Arizona, Tucson, AZ 85721 USA, on leave with University of the West of England, Faculty of Computing, Engineering and Mathematical Sciences, Bristol, U.K. (e-mail: ivan@ece.arizona.edu).

B. Vasic is with Department of Electrical and Computer Engineering, University of Arizona, Tucson, AZ 85721 USA (e-mail: vasic@ece.arizona.edu).

J. Rorison is with Department of Electrical and Electronic Engineering, University of Bristol, Bristol BS8 1TR, U.K. (e-mail: Judy.Rorison@bristol.ac.uk).

Digital Object Identifier 10.1109/LCOMM.2004.825709

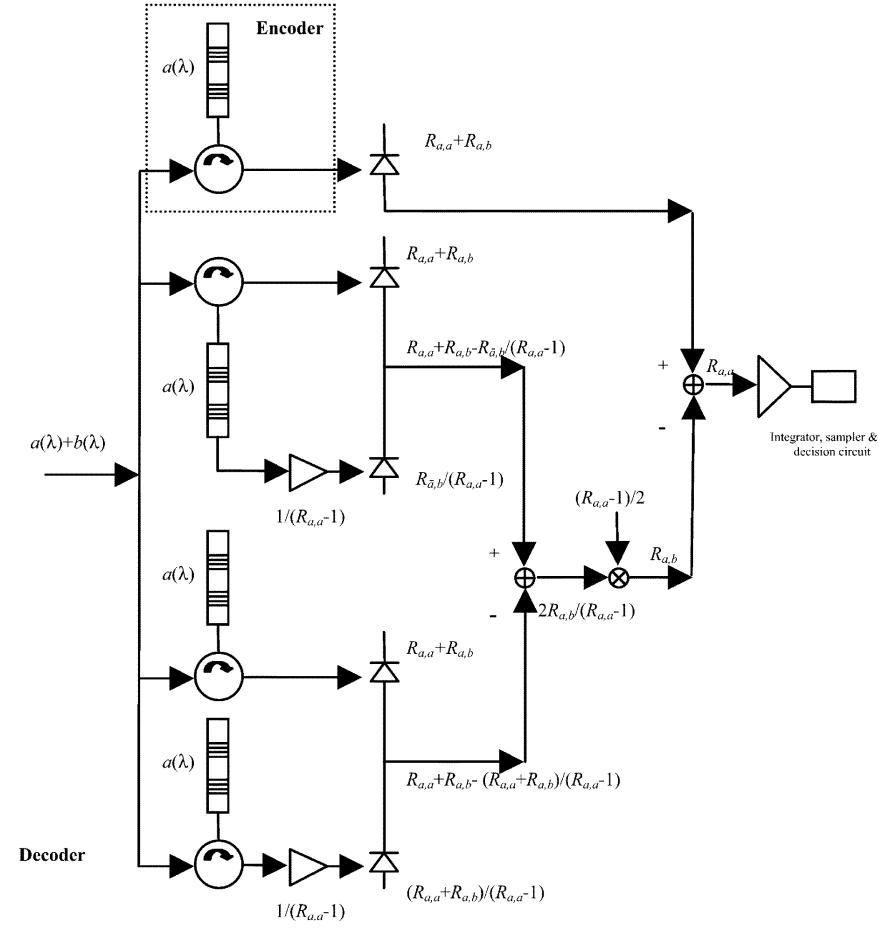

Fig. 1. MAI cancellation decoder scheme $\left(R_{a, a}\right.$-the weight of codeword $\left.a\right)$.

designs (PBD). The algorithm to construct PBD optical orthogonal codes (OOCs) from resolvable balanced incomplete block designs (BIBDs) supporting arbitrary many different services is described. It is illustrated on our novel combinatorial construction-the integer lattice construction.

\section{ENCODER/DECODER STRUCTURE}

Let $a=\left(a_{1}, a_{2}, \ldots, a_{\nu}\right)$ and $b=\left(b_{1}, b_{2}, \ldots, b_{\nu}\right)$ be two different code sequences. Their mutual in-phase cross correlation is defined as $R_{a, b}=\sum_{n=1}^{\nu} a_{n} b_{n}$. Unipolar OOCs having fixed in-phase cross correlation are the only codes considered so far for application in SAC optical CDMA, as they require a simple balanced detection scheme (proposed in [3]) to eliminate MAI. The sequences with nonfixed in-phase cross correlation do not satisfy the condition $R_{\bar{a}, b}=R_{a, b}$, and the balanced detection scheme [3] cannot be applied. Those sequences satisfy the relationship

$$
R_{\bar{a}, b}+R_{a, b}=R_{b, b}
$$

with $R_{b, b}=b_{1}+b_{2}+\ldots+b_{\nu}$ being the codeword weight.

We propose novel, fiber-Bragg grating based, balanced detection scheme that allows the MAI cancellation in the case when 
TABLE I

A LatTICE $2-(28,\{1,3,4,5\},\{0,1\})$ PBD DESIGN

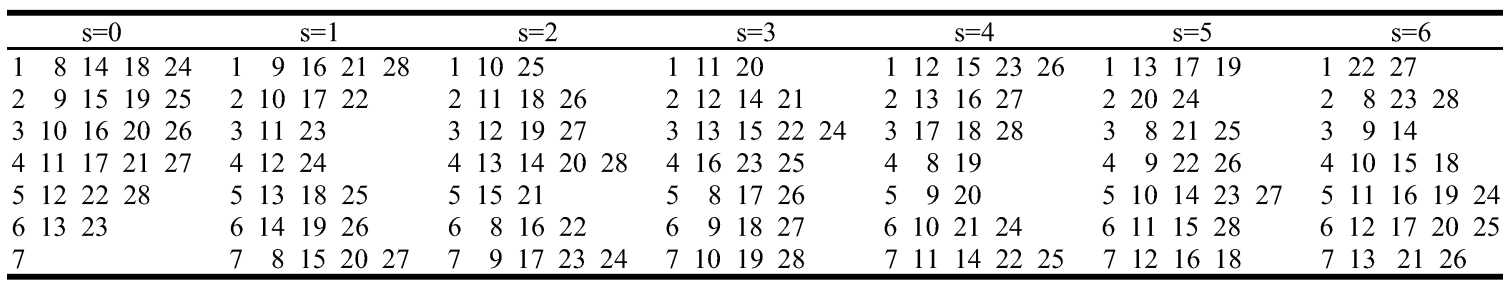

unipolar sequences have limited but not fixed in-phase cross correlation. More specifically, our scheme requires $R_{a b} \leq 1$. Since the in-phase cross correlation constraint is relaxed (not-fixed), the resulting OOC has larger cardinality (as it will be shown in Section III). The transmitter and receiver structures based on FBG's are shown in Fig. 1.

When bit " 1 " is sent, an optical pulse from a broadband source is launched into encoder, while no optical pulse is launched for data bit "0". The optical pulse passes through the linear FBG array in encoder and spectral components with the spectral distribution $a(\lambda)$ are reflected. At the receiver, each grating is fixed according to receiver's address. For proper decoding, the peak wavelengths are arranged in opposite order so that round-trip delays of different spectral components are compensated. The lower decoder branch, i.e. the dual-balanced detection branch, is used to estimate the MAI, and its output is proportional to $R_{a, b}$, while the upper branch is the signal-detection brunch whose output is proportional to $R_{a, a}+R_{a, b}$ (the signal values at all other points are shown in Fig. 1.) Therefore, the detector scheme shown in Fig. 1, completely cancels MAI.

\section{PAIRwise BAlanced Design BASEd OOC CONSTRUCTIONS}

In Section II we introduced a balanced detector that can be used for a nonfixed in-phase cross correlation OOC's. The motivation is that the cardinality of the OOC, and therefore the number of simultaneous users, can be increased. The problem of constructing OOC family with such properties for multimedia applications is presented in this section. It is based on combinatorial objects referred to as pairwise balanced designs constructed from the balanced incomplete block designs.

A balanced incomplete block design (BIBD) is defined as a collection of $k$-subsets of a $v$-set $V, k<v$, such that every pair of elements of $V$ occurs together in exactly $\lambda$ of the blocks. Each $k$-subset is called a block and each element of $V$ is called a point. The design is said to be balanced because each pair of points from $V$ occur together in exactly $\lambda$ of the blocks, and it is said to be incomplete because not all possible $k$-blocks of $V$ are elements of the blocks $\lambda$ set. A BIBD is referred to as a design with parameters $2-\operatorname{BIBD}(v, k, \lambda)$. (For more details about designs and BIBDs interested reader is referred to [8].) If we further allow different sizes of the blocks in a design a pairwise balanced design (PBD) results. Therefore, a PBD is defined as a collection of blocks of different sizes taken from $K$, such that every pair of points is contained in $\lambda$ of the blocks, and it is denoted as $\operatorname{PBD}(v, K, \lambda)$.
Now we address the problem of constructing PBDs from BIBDs. We present two constructions. The first one is a based on a general theory of PBDs [8], and the second one uses affine sub-geometry on an integer lattice. Notice first, that the methods proposed so far (e.g., [4], [5]) support two different multimedia services only, as their OOC families contain the codewords of only two different weights. For our first construction we use the PBD construction methods given in [8]. Actually, [8] gives the following three methods allowing the construction of a PBD from a BIBD: 1) the method of adjoining elements; 2) the method of removing elements; and 3) difference systems based constructions. Such PBD OOC families support up to four different services. In order to accommodate larger number of services, we propose the following approach based on resolvable BIBDs. Let $B=\left(b_{i j}\right)$ be an array of blocks within the first resolvability class, and let $b_{i j}(1 \leq i \leq m, 1 \leq j \leq k$, $m$-the number of blocks in the resolvability class) denote the points of $i$ th block. Let $\beta=\left[\beta_{1} \beta_{2} \ldots \beta_{k}\right]$ be the "population vector" with elements denoting the percentage of points from $j$ th $(1 \leq j \leq k)$ column remaining undeleted. Delete the last $\left\lfloor m\left(1-\beta_{j}\right)\right\rfloor(\lfloor x\rfloor$ denotes an integer part of a real number $x)$ points from $j$ th $(1 \leq j \leq k)$ column and renumerate the remained points. Deleting points does not violate the constraint that the pair of points is contained in at most one block. The algorithm can be used on any resolvable BIBD.

In the next section we present a novel construction of OOC codes and then demonstrate our proposal to construct sequences of different weights.

\section{INTEGER LATTICE CONSTRUCTION OOC}

The second construction is based on a points and lines on a subset of a rectangular integer lattice. The subsets of points (but not all subsets) are referred to as lines, and the design is defined as a set of lines of different slopes. A line with slope $s, 0 \leq s \leq m-1$, starting at the point $(x, a)$, contains the points $\{(x, a+s x \bmod m): 0 \leq x \leq k-1\}$, where $0 \leq$ $s \leq m-1$. The total number of lines is equal to $m^{2}$. Therefore, the maximum number of users is $m^{2}$, the codeword length is $m \cdot k$ and the codeword weight $k$. It can be readily verified that arbitrary large families are possible as long as $m$ and $k$ are coprime.

The integer lattice construction with vertical and horizontal dimensions being 7 and 5, respectively; and population vector $\left[\begin{array}{lllll}1 & 0.8 & 0.5 & 0.8 & 0.7\end{array}\right]$ yields to the OOC family having 14 users of weight 5,23 users of weight 4,11 users of weight 3 , and one user of weight 1 . The multi-weight OOC codewords are given in Table I, with labels being the positions of ones within a 


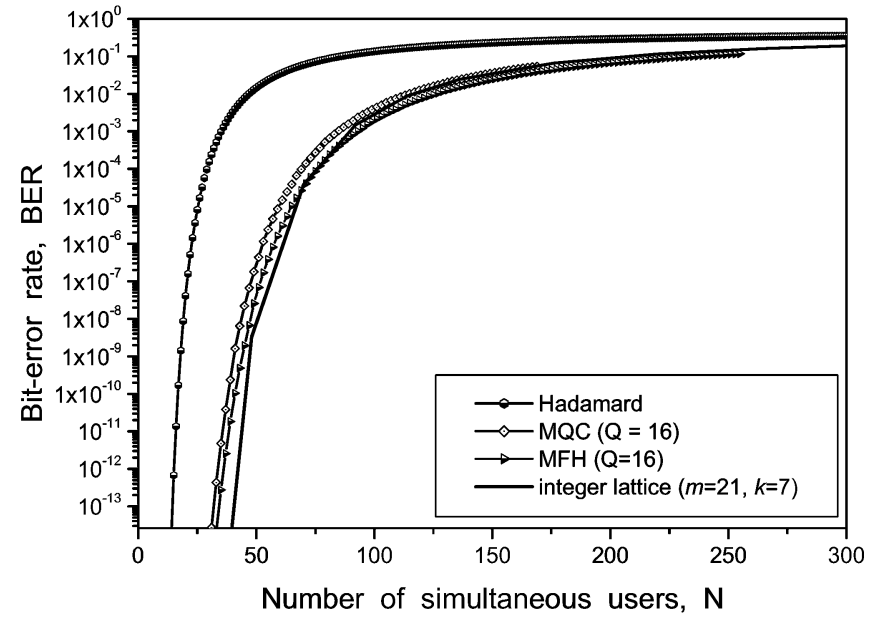

Fig. 2. Single-weight integer lattice code against MFH and MQC.

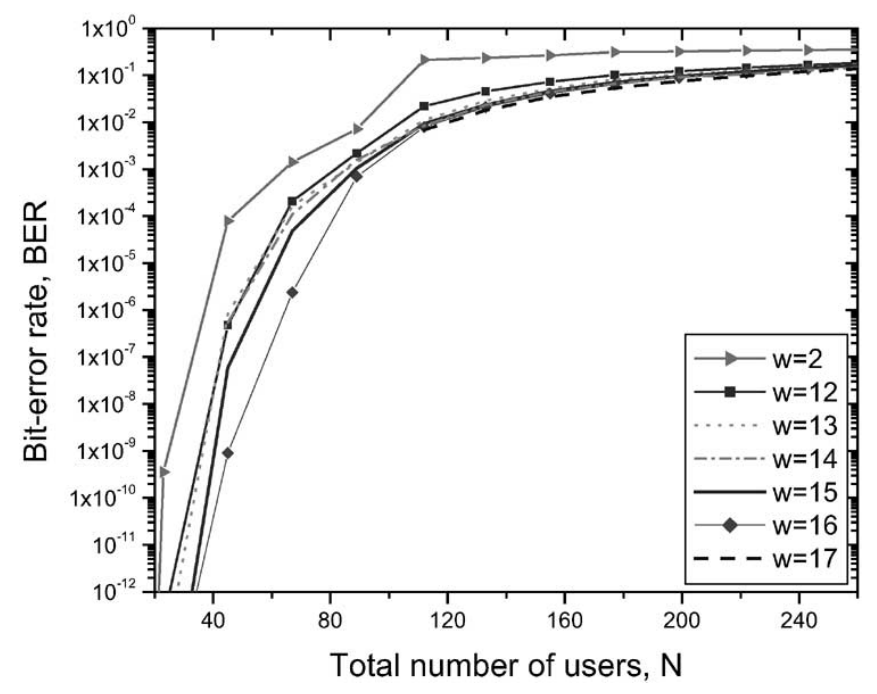

Fig. 3. Multi-weight OOC performance for SAC optical CDMA system based on integer lattice $\mathrm{PBD}, m=21, k=17$, with population vector $\left[\begin{array}{lllll}1 & 0.9 & \ldots & 0.9 & 1\end{array}\right]$.

codeword. Namely, the population vector indicates that the last point in the second column $\left(\left\lfloor m\left(1-\beta_{2}\right)\right\rfloor=\lfloor 7(1-0.8)\rfloor=1\right)$, the last three points in the third, the last point in the fourth and the last two points in the fifth column of the point-block matrix of the initial design, corresponding to the lines of slope $s=0$, are to be removed. After renumeration of the remained points, the resulting design in Table I is obtained.

\section{PERformance AnAlysis}

The proposed single-weight integer lattice against previously reported MFH [2], MQC [2], and Hadamard code [1] in the presence of the phase induced intensity noise, the photodiode shot noise and the thermal noise is illustrated in Fig. 2.

The system parameters are: the optical source with uniform spectrum and bandwidth $\Delta \nu=3.75 \mathrm{THz}$, the electrical equivalent receiver noise bandwidth $B=311 \mathrm{MHz}$, the bit-rate $622 \mathrm{Mb} / \mathrm{s}$, the central wavelength $1550 \mathrm{~nm}$, the receiver noise absolute temperature $T_{r}=300 \mathrm{~K}$, the load resistance $R_{L}=$ $1030 \Omega$, the photodiode quantum efficiency 0.6. Notice that BER performance of integer lattice OOC for $m=21, k=7$ is comparable or better than MFH, although the code weight and lengths are significantly smaller, and supports larger number of users. Namely, the cardinality of MFH OOC is $q^{2}=256$ (where $q$ is a prime power), while that in integer lattice design is $m^{2}=441$. MFH code weight and lengths are $q=16$ and $q^{2}+q=272$, respectively; while the integer lattice codeword weight and length are $k=7$ and $m k=147$, respectively. Similarly to [1]-[3] the Gaussian approximation is applied and method of averaging the cross correlations (similarly to [7]), so that the average service BER performance is obtained.

The performance of the multi-weight lattice construction is illustrated in Fig. 3 for vertical and horizontal dimensions 21 and 17 and population vector $\left[\begin{array}{llll}1 & 0.9 \ldots 0.9 & 1\end{array}\right]$. The construction support maximum 4 users of weight 2, 36 users of weight 12, 96 users of weight 13, 120 users of weight 14, 132 users of weight 15, 12 users of weight 16 and 41 users of weight 17, and therefore, seven different services may be implemented. (In the calculation an equal fraction of sequences from different services is assumed.)

By changing the population vector many different multi-weight classes can be generated.

\section{CONCLUSION}

A novel class of constant-length variable-weight optical orthogonal codes suitable to multimedia services with different quality-of-service requirements is proposed in this letter. The construction is based on the pairwise balanced designs. The construction algorithm is simple and it is illustrated on our novel construction of OOC, the integer lattice. The previous constructions [1]-[3] mainly have fixed in-phase cross correlation and there are not applicable in multimedia applications. In integer lattice construction the codewords have in-phase cross correlation 0 or 1 , many codewords are orthogonal to each other, which enabled us to obtain larger families. The novel balanced detection FBG scheme is also proposed. It cancels the MAI in multimedia environment in a SAC scheme employing sequences having the nonfixed in phase cross correlation.

\section{REFERENCES}

[1] E. D. J. Smith, R. J. Blaikie, and D. P. Taylor, "Performance enhancement of spectral-amplitude-coding optical CDMA using pulse-position modulation," IEEE Trans. Commun., vol. 46, pp. 1176-1185, Sept. 1998.

[2] Z. Wei and H. Ghafouri-Shiraz, "Unipolar codes with ideal in-phase cross-correlation for spectral amplitude-coding optical CDMA systems," IEEE Trans. Commun., vol. 50, no. 8, pp. 1209-1212, 2002.

[3] Z. Wei et al., "Modified quadratic congruence codes for fiber Bragg-grating-based spectral-amplitude-coding optical CDMA systems," J. Lightwave Technol., vol. 19, pp. 1274-1281, Sept. 2001.

[4] W. C. Kwong and G.-C. Yang, "Double-weight signature pattern codes for multicore-fiber code-division multiple-access networks," IEEE Commun. Lett., vol. 5, pp. 203-205, May 2001.

[5] - "Design of multilength optical orthogonal codes for optical CDMA multimedia networks," IEEE Trans. Commun., vol. 50, pp. 1258-1265, Aug. 2002.

[6] J.-G. Zhang, "Flexible optical fiber CDMA networks using strict optical orthogonal codes for multimedia broadcasting and distribution applications," IEEE Trans. Broadcasting, vol. 45, pp. 106-115, Mar. 1999.

[7] S. V. Maric, O. Moreno, and C. J. Corrada, "Multimedia transmission in fiber-optic LAN's using optical CDMA," J. Lightwave Technol., vol. 14, pp. 2149-2153, Oct. 1996.

[8] C. J. Colbourn and J. H. Denitz, The CRC Handbook of Combinatorial Designs. Boca Raton, FL: CRC Press, 1996. 